\title{
Low-voltage solution-processed hybrid light-emitting transistors
}

Mujeeb Ullah Chaudhry ${ }^{* 1}$, Kornelius Tetzner ${ }^{2}$, Yen-Hung Lin ${ }^{4}$, Sungho Nam ${ }^{4}$, Christopher Pearson $^{1}$, Chris Groves ${ }^{1}$, Michael C. Petty ${ }^{1}$, Thomas D. Anthopoulos ${ }^{* 2,3}$, Donal D. C. Bradley $^{* 4,5}$

${ }^{1}$ Department of Engineering, Durham University, Durham, DH1 3LE, United Kingdom

${ }^{2}$ Blackett Laboratory, Department of Physics and Centre for Plastic Electronics, Imperial College London, London SW7 2BW, United Kingdom

${ }^{3}$ Physical Science and Engineering Division, King Abdullah University of Science and Technology, Thuwal 23955, Saudi Arabia

${ }^{4}$ Department of Physics, University of Oxford, Oxford OX1 3PU, United Kingdom

${ }^{5}$ Department of Engineering Science, University of Oxford, Oxford OX1 3PJ, United Kingdom

* Correspondence: mujeeb.u.chaudhry@ durham.ac.uk (MUC), thomas.anthopoulos@kaust.edu.sa (TDA), donal.bradley@mpls.ox.ac.uk (DDCB)

\begin{abstract}
We report the development of low operating voltages in inorganic-organic hybrid lightemitting transistors (HLETs) based on solution-processed $\mathrm{ZrO}_{\mathrm{x}}$ gate dielectric and a hybrid multilayer channel consisting of the heterojunction $\operatorname{In}_{2} \mathrm{O}_{3} / \mathrm{ZnO}$ and the organic polymer 'Super Yellow' acting as n- and p-channel/emissive layers, respectively. Resulting HLETs operate at the lowest voltages reported to-date $(<10 \mathrm{~V})$ and combine high electron mobility $\left(22 \mathrm{~cm}^{2} / \mathrm{Vs}\right)$ with appreciable current on/off ratios $\left(\approx 10^{3}\right)$ and an external quantum efficiency of $2 \times 10^{-2} \%$ at $700 \mathrm{~cd} / \mathrm{m}^{2}$. The charge injection, transport and recombination mechanisms within this HLET architecture are discussed and prospects for further performance enhancement are considered.
\end{abstract}

\section{Keywords}

Light emitting transistors; low-voltage; solution-processed organic semiconductors; hybrid transistors, metal oxide high-k dielectric 
Solution processable semiconductor and dielectric materials are set to play an important role in future electronic and optoelectronic technologies due to the ability to form high quality thin films by cost-efficient, solution-based manufacturing processes ${ }^{1-7}$. Recently, solution-processed hybrid heterostructure (organic/inorganic) light emitting transistors (HLETs) containing an n-type metal oxide layer (acting as the electron transporting channel) and a conjugated polymer layer (acting as the light-emitting layer) have demonstrated significant potential for novel display applications ${ }^{8-12}$. Since HLETs provide gate controlled light emission, they can, potentially, further simplify the fabrication of display pixel devices $^{11,12}$. However, the operating voltages for best performing HLETs have remained stubbornly high $(\approx 100 \mathrm{~V})$, hindering the demonstration of power-efficient devices. Further to high operating voltages, Si substrates, used in reported HLETs, absorb the emitted light and result in lower optical performance in comparison to similar structure organic light emitting diodes (OLEDs) ${ }^{13-15}$ (where the metal cathode reflects light out through a transparent anode). Thus the development of low-operating-voltage HLETs incorporating solution-processed materials on non-absorbing substrates is a necessary step towards meeting the challenges for next-generation low-cost display applications. An interesting approach that can help to address this need is the use of high-k dielectric materials in combination with Al-gate electrodes in novel device architectures ${ }^{16-19}$.

It has recently been shown that the charge carrier mobility in solution-processed metal oxide transistors can be enhanced by the introduction of low-dimensional heterostructure channel systems with carefully engineered interface energetics ${ }^{1,2,17-21}$. It has been argued that due to the conduction band energy offset between the two oxides employed, electrons migrate towards the hetero-interface forming a quasi-two-dimensional (2D) free-electron 
system which in turn leads to remarkable improvements in the electron mobility of the device. Depending on various factors such as complexity of the heterostructure, deposition method and annealing temperature, electron mobility values up to $50 \mathrm{~cm}^{2} / \mathrm{Vs}$ have been attained $\mathrm{d}^{10,11}$. Furthermore, it was found that the use of low temperature, solution processed and high-k dielectrics, i.e. $\mathrm{ZrO}_{\mathrm{x}}$ (dielectric constant 25), is able to increase the charge carrier mobility of such heterostructure channel systems whilst offering a dramatic reduction in transistor operating voltage ${ }^{16-18}$. Similar advantages are found in using high- $\mathrm{ZrO}_{\mathrm{x}}$ dielectrics in $\mathrm{ZnO}$ and $\mathrm{Li}$-doped $\mathrm{ZnO}$ transistors ${ }^{22}$ and $\mathrm{ZrO}_{\mathrm{x}}$ is also known to function effectively as an electron injection layer in hybrid light emitting diodes ${ }^{23}$.

Herein, we report the development of low-voltage HLETs fabricated using a combination of solution-processable organic and inorganic materials with carefully selected functionalities. In particular, by integrating ultra-thin layers of the high- $k$ dielectric $\mathrm{ZrO}_{\mathrm{x}}$ with an n-channel $\mathrm{In}_{2} \mathrm{O}_{3} / \mathrm{ZnO}$ heterojunction and the p-channel highly luminescent polymer Super Yellow (SY), we are able to demonstrate low operating voltage ( $\leq 10 \mathrm{~V})$ HLETs. Additionally, the resulting hybrid devices offer improved electroluminescence characteristics with excellent transistor operation that includes high electron mobility $\left(\approx 22 \mathrm{~cm}^{2} \mathrm{~V}^{-1} \mathrm{~s}^{-1}\right)$ and appreciable ON/OFF current ratios. These attributes enable relatively high channel currents to be driven through the active layer of the HLET leading to a luminance of up to $700 \mathrm{~cd} / \mathrm{m}^{2}$, albeit at a modest $\mathrm{EQE} \sim 10^{-2} \%$.

The HLETs were fabricated on pre-cleaned glass substrates, employing a $50 \mathrm{~nm}$-thick $\mathrm{Al}$ gate electrode deposited via thermal evaporation. A thin layer of native $\mathrm{Al}_{2} \mathrm{O}_{3}$ was grown by exposing the $\mathrm{Al}$ electrodes to ozone generated by a low-pressure mercury UV lamp. The $\mathrm{ZrO}_{\mathrm{x}}$ precursor solution was then spin-coated on top under $\mathrm{N}_{2}$, followed by a photochemical 
curing step in ambient air (as shown in Figure S1 in Supporting Information). The surface topography of each of the sequentially deposited layers was characterized using atomic force microscopy (AFM) (Figure 1 (a-d)). The AFM images (Figure 1 (a-c)) show that the thermally evaporated Al electrodes feature a relatively high root mean square surface roughness $\left(\mathrm{R}_{\mathrm{RMS}}\right.$ ) of $\sim 1.2 \mathrm{~nm}$, which increases to $1.4 \mathrm{~nm}$ following $\mathrm{Al}_{2} \mathrm{O}_{3}$ formation. Interestingly, solution-deposition of the $\mathrm{ZrO}_{\mathrm{x}}$ layer appears to planarize the $\mathrm{Al} / \mathrm{Al}_{2} \mathrm{O}_{3}$ surface as evidenced by the reduced surface $\mathrm{R}_{\mathrm{RMS}}=0.7 \mathrm{~nm}$. Figure $\mathbf{1}(\mathbf{d})$ shows the corresponding surface height distributions for each sample, where the labels $\mathrm{Al}, \mathrm{AlOx}$ and $\mathrm{ZrOx}$ refer to the top layer in the stack, i.e. the $\mathrm{ZrOx}$ data represents the surface of the $\mathrm{Al} / \mathrm{AlOx} / \mathrm{ZrOx}$ gate electrode/dielectric stack. Figure 1(e) shows the capacitance versus frequency (C-f) characteristics of the $\mathrm{Al}_{2} \mathrm{O}_{3} / \mathrm{ZrO}_{\mathrm{x}}$ bilayer dielectrics measured in a metal/insulator/metal (MIM) configuration (see inset in Figure 1(e)). Analysis of the C- $f$ data allows calculation of the geometrical capacitance of the gate dielectric, yielding a value of $\approx 235 \mathrm{nF} / \mathrm{cm}^{2}$. The breakdown electric field and leakage current is shown in Figure S2 in the Supporting

\section{Information.}

For the n-channel, the $\operatorname{In}_{2} \mathrm{O}_{3}$ layer was subsequently grown on the dielectric by spincoating a precursor solution (see section Materials and Methods in Supporting Information) in air followed by thermal annealing at $300{ }^{\circ} \mathrm{C}$ (also in air), repeated three times in order to increase thickness. The top $\mathrm{ZnO}$ layer was then deposited by spin-coating a nanoparticle solution and thermal annealing at $300{ }^{\circ} \mathrm{C}$ in air. HLET fabrication was completed using two shadow-masked evaporation steps to define complementary electronand hole-injecting source (S) and, respectively, drain (D) contacts with an intervening emission layer deposition step resulting in a non-planar S/D geometry (Figure 2(a)). The electron-injecting Al S-electrode was first deposited directly on top of the $\operatorname{In}_{2} \mathrm{O}_{3} / \mathrm{ZnO}$ 
heterojunction. Then a $120 \mathrm{~nm}$ thickness layer of the emission polymer, Super Yellow (molecular structure as shown in Figure S3 in Supporting Information), ${ }^{24}$ was spin-coated over the whole structure and annealed at $150{ }^{\circ} \mathrm{C}$ in nitrogen atmosphere. Finally, the $\mathrm{MoO}_{\mathrm{X}} / \mathrm{Au}$ hole-injecting D-electrode was evaporated to complete the non-planar S-D geometry $^{25-26}$. The channel width and length of the resulting HLETs was measured to be 1 $\mathrm{mm}$ and $100 \mu \mathrm{m}$, respectively. The details of all fabrication steps are given in the section, "Materials and Methods" in Supporting Information and a schematic illustration of each dielectric layer and electrode deposition is depicted in Fig. S1 and S4 respectively (Supporting Information).

Figure 2 parts (b) and (c) show the transfer and output characteristics of a representative device. The high capacitance of the $\mathrm{Al}_{2} \mathrm{O}_{3} / \mathrm{ZrO}_{\mathrm{x}}$ bilayer dielectric allows these transistors to be operated at $\leq 10 \mathrm{~V}$ bias and they exhibit clear n-channel behavior with no operating hysteresis between forward and return gate voltage $\left(V_{G}\right)$ sweeps for both linear and saturation regimes. An average electron mobility of $\approx 22 \mathrm{~cm}^{2} \mathrm{~V}^{-1} \mathrm{~s}^{-1}$ was calculated from the transfer curves measured in saturation regime using the equation (1), in agreement with previous reports for similar transistor channel architectures ${ }^{1-5}$.

$$
I_{D S}=\frac{W C_{i}}{2 \times L} \mu\left(V_{G S}-V_{T H}\right)^{2}
$$

where $I_{D S}$ is the experimentally measured drain-source current, $W / L$ is the width-to-length ratio of the device channel, $V_{G S}$ is the operating gate voltage and $C_{i}$ is the geometric capacitance of the bilayer dielectric. The electron mobility was calculated from the high voltage slope of the transfer characteristics as shown in Figure S5 in Supporting Information. We further analyzed the electron mobility to calculate the reliability factor ${ }^{28}$ of $195 \%$ and effective mobility of $43 \mathrm{~cm}^{2} \mathrm{~V}^{-1} \mathrm{~s}^{-1}$ (explained and given in Table $\mathbf{S 1}$ in Supporting Information). The device also exhibits an appreciable on/off channel current 
ratio $\approx 5 \times 10^{2}$. These results represent the lowest operating voltage and highest electron mobility reported to date for a solution-processed HLET structure.

Another observation concerns the non-linear channel current (IDS) increase at low drain voltage $\left(\mathrm{V}_{\mathrm{DS}}\right)$ seen in Figure 2(c). We attribute this to contact resistance, which although relatively low in absolute terms is nevertheless significant in the context of our high charge carrier mobility semiconductor. The contact resistance is also visible in the sigmoidal output characteristics given in Figure S6 in Supporting Information. We note that further improvements should then be realizable through additional contact resistance optimization. The contact resistance from the hole injecting electrode (MoOx/Au) in inevitable in the HLETs and cannot be avoided. However, to lower the electron injection barrier into SY films from $\mathrm{ZnO} / \mathrm{In}_{2} \mathrm{O}_{3}$ layer, a work function modifying interlayer material (e.g. $\mathrm{Cs}_{2} \mathrm{CO}_{3}$ or PEIE) can be used as reported previously ${ }^{11-12}$.

The electrical and optical characteristics of the devices were tested within a nitrogenfilled glove box using an Agilent B2902A semiconductor parameter analyzer and a probestation fitted with a calibrated photodiode positioned over the active region of the device according to our previously reported method ${ }^{25-27}$. External quantum efficiencies, EQEs, were calculated from the luminance, source-drain current and emission spectra of the devices under the assumption of Lambertian emission as reported previously ${ }^{25-27}$ (full detail of electrical and optical characterization is given in Supporting Information). Figure 3(a) shows the current density and luminance plotted as a function of the applied gate voltage. Current density here $\left(\mathrm{mA}-\mathrm{A} / \mathrm{cm}^{2}\right)$ is calculated from the field-effect channel current density $\left(\mathrm{A}-\mathrm{kA} / \mathrm{cm}^{2}\right)$ taking into account the observation that the current contributing to emission is spread over the entire recombination zone area (width $12 \mu \mathrm{m} \times$ length $2 \mathrm{~mm}$ ) rather than 
being confined only to the charge accumulation layer as described earlier ${ }^{8-12,29}$. At $\mathrm{V}_{\mathrm{DS}}=10$ $\mathrm{V}$ and $\mathrm{V}_{\mathrm{GS}}=10 \mathrm{~V}$ we find $\mathrm{J}=2 \mathrm{~A} / \mathrm{cm}^{2}$. Figure 3(b) reports the external quantum efficiency, EQE, $(\%)$ as a function of luminance $\left(\mathrm{cd} / \mathrm{m}^{2}\right)$ for a typical HLET; the inset shows an optical micrograph of the HLET channel during operation with visible light-emission emanating from close to the $\mathrm{MoO}_{\mathrm{X}} / \mathrm{Au}$ D-electrode. This suggests that unbalanced injection/transport is playing a key role with an excess of electrons accumulating in the channel and injected into the SY film close to the D-electrode. A maximum luminance of $700 \mathrm{~cd} / \mathrm{m}^{2}$ is achieved at the highest investigated drain current $\left(2 \mathrm{~A} / \mathrm{cm}^{2}\right)$ and gate voltage $(10 \mathrm{~V})$. An interesting feature is the observed saturation of the calculated EQE at a maximum value close to $2 \times 10^{-2} \%$. The latter value is similar to that reported previously for HLETs operated at significantly higher gate voltages $(\approx 100 \mathrm{~V}) .{ }^{8-11}$ The light emission zone also did not shift with $\mathrm{S}$-D voltage appearing stationary at the hole-injecting electrode, again characteristic of unipolar, or heavily unbalanced charge transport. The optical micrograph of the working device shown in the inset to Figure 3b was further analyzed (Figure S7, Supporting Information), yielding an emission zone width of $12( \pm 2) \mu \mathrm{m}$. The electroluminescence (EL) and photoluminescence (PL) spectra of the HLET are shown in Figure 4(a) and accord with the expected emission from $\mathrm{SY}^{24-26}$. EL was recorded for $\mathrm{V}_{\mathrm{DS}}=10 \mathrm{~V}, \mathrm{~V}_{\mathrm{G}}=10 \mathrm{~V}$ and $\mathrm{J}_{\mathrm{DS}}=2 \mathrm{~A} / \mathrm{cm}^{2}$ and PL under optical excitation at $365 \mathrm{~nm}$ using a LED source. We note that since the $\mathrm{MoO}_{\mathrm{x}} / \mathrm{Au} \mathrm{D}-$ electrode is not fully transparent (Figure S8, Supporting Information), the observed light is most likely limited to photons emitted from around the edges of the electrode, with additional exciton generation and emission expected to occur beneath it. This will lead to an underestimate of the emission efficiency, in agreement with previous reports $8,11,26$. However, it is worth mentioning that the HLET architecture employing the Al bottom-gate electrode exhibit higher EQE than previously reported HLETs fabricated on $\mathrm{Si}^{++}$substrates ${ }^{8}$. We attribute this to reflection of the emitted light by the bottom Al gate through the 
semitransparent top drain electrode. The overall result is an increase in the measured EQE. In contrast, most of the previously reported HLETs were fabricated on $\mathrm{Si}^{++}$substrates where a fraction of the emitted photons are absorbed by the $\mathrm{Si}^{++}$, resulting in a lower EQE. ${ }^{8-12}$ The advantages of the proposed HLET architecture over previously reported HLETs are summarized in Table S2 in the Supporting Information.

HLET operation therefore involves the following four steps; (i) electron injection from the Al S-electrode into the $3.8-4.0 \mathrm{eV}^{18}$ conduction band of the $\operatorname{In}_{2} \mathrm{O}_{3} / \mathrm{ZnO}$ heterostructure, (ii) transport of electrons along the $\operatorname{In}_{2} \mathrm{O}_{3} / \mathrm{ZnO}$ interface, (iii) accumulation of electrons at the interface between $\mathrm{In}_{2} \mathrm{O}_{3} / \mathrm{ZnO}$ and $\mathrm{SY}$, and (iv) recombination of these electrons with holes injected from the $\mathrm{MoO}_{\mathrm{x}} / \mathrm{Au}$ D-electrode into the highest occupied molecular orbital (HOMO) of SY $(5.3 \mathrm{eV}) .{ }^{25-26}$. From the schematic energy level diagram in Figure 4(b) and the above discussion it seems likely that an electron injection interlayer could be used to help overcome the large electron injection barrier between the conduction band of $\operatorname{In}_{2} \mathrm{O}_{3} / \mathrm{ZnO}$ and the lowest unoccupied molecular orbital (LUMO) of Super Yellow ${ }^{25-26}$ and that this should lead to higher efficiency and a more uniform emission zone. Further studies will address this consideration.

In summary, low operating voltage hybrid light-emitting transistors were successfully demonstrated using a stack channel architecture composed of a solution-processed metal oxide dielectric, a solution-processed heterojunction metal oxide n-channel and a conjugated light-emitting polymer. Resulting devices exhibit clear n-channel transistor characteristics with a high electron mobility of $\approx 22 \mathrm{~cm}^{2} / \mathrm{Vs}$. Under normal operating conditions, the devices emit bright yellow light, characteristic of the SY polymer used. Quantitative analysis of the light-emission yields a device luminance of up to $700 \mathrm{~cd} / \mathrm{m}^{2}$ at a maximum EQE of $2 \times 10^{-2} \%$. 
These are the lowest-operating-voltage HLETs reported to date and highlight the potential of our multilayer hybrid channel architecture.

\section{Supporting Information:}

Materials and Methods, Device fabrication: dielectrics layers deposition, Breakdown electric filed for the dielectric stack and device leakage current, Chemical structure of the emissive polymer PD-132, "Super Yellow", Device fabrication: electrodes and emissive layer deposition, Reliability factor and effective mobility calculation, Linear fit to the SQRT (IDS) vs. VGS and effective mobility, Electrical and optical output characteristics, Microscopic image intensity profile of the HLET channel and electrodes, Transmission of hole injecting $\mathrm{MoOx} / \mathrm{Au}$ electrode, Comparison of charge carrier mobilities and operating voltages in HLETs.

\section{Acknowledgement:}

This work is supported by a Durham Junior Research Fellowship COFUNDed between Durham University and the European Union (grant agreement no. 609412). MUC, CP, CG and MCP thank Institute of Advanced Study, Durham University for their Support. KT, YHL and TDA. acknowledge financial support from the People Programme (Marie Curie Actions) of the European Union's Framework Programme Horizon 2020: “Flexible Complementary Hybrid Integrated Circuits"' (FlexCHIC), grant agreement $N^{\circ} 658563$. DDCB thanks the University of Oxford for start-up funding (grant no. DK3004), including a postdoctoral research fellowship for SN. 

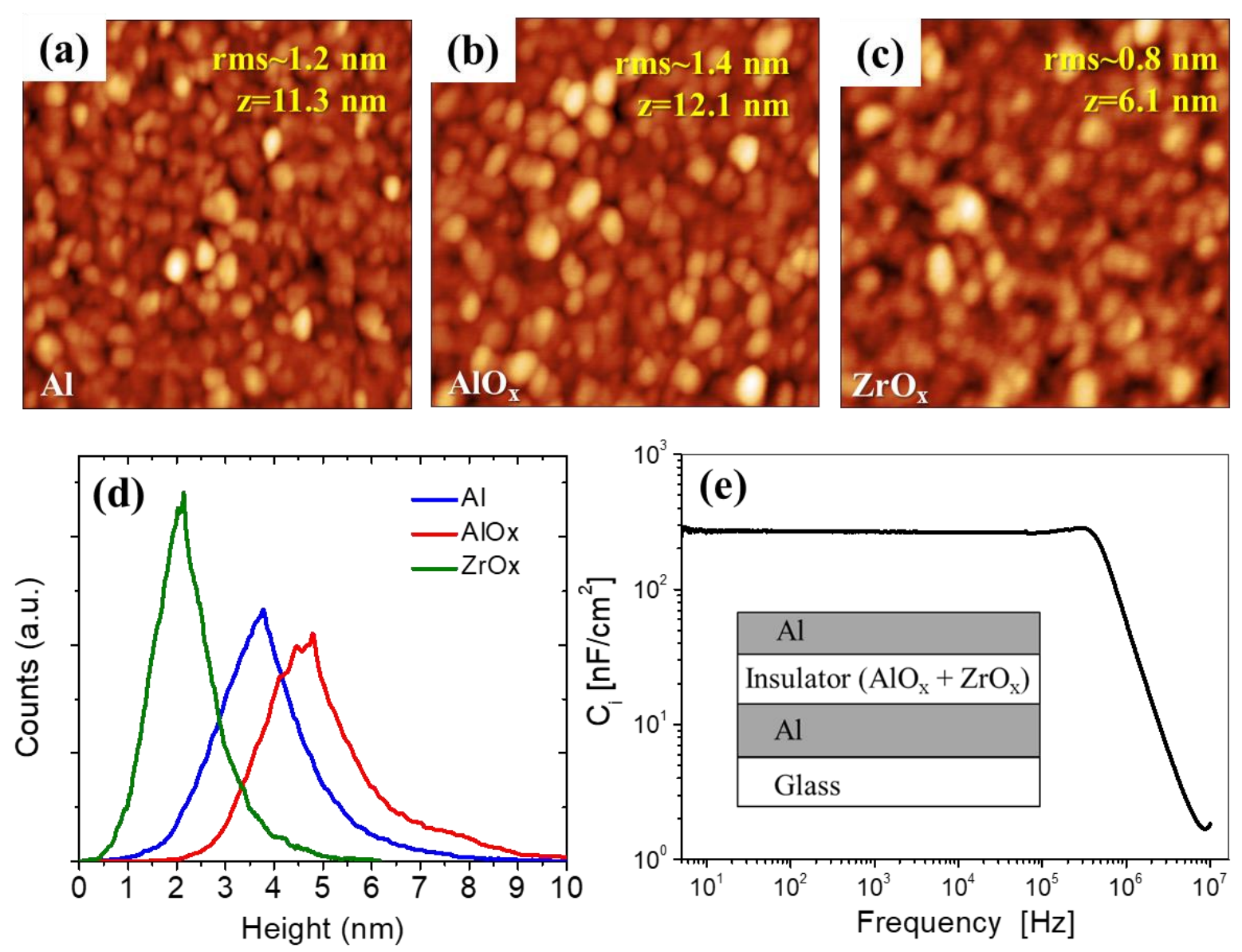

Figure 1. Sequential atomic force micrograph images $(1 \mu \mathrm{m} \times 1 \mu \mathrm{m})$ of the top surface of (a) $\mathrm{Al}$, (b) $\mathrm{Al} / \mathrm{AlO}_{\mathrm{x}}$, (c) $\mathrm{Al} / \mathrm{AlO}_{\mathrm{x}} / \mathrm{ZrO}_{\mathrm{x}}$ layers and (d) height distributions for these images. (e) Capacitance $v s$ frequency characteristics of the solution processed $\mathrm{AlOx} / \mathrm{ZrO}_{\mathrm{x}}$ bilayer dielectrics; the inset shows the metal/insulator/metal device structure used for capacitance measurements. 

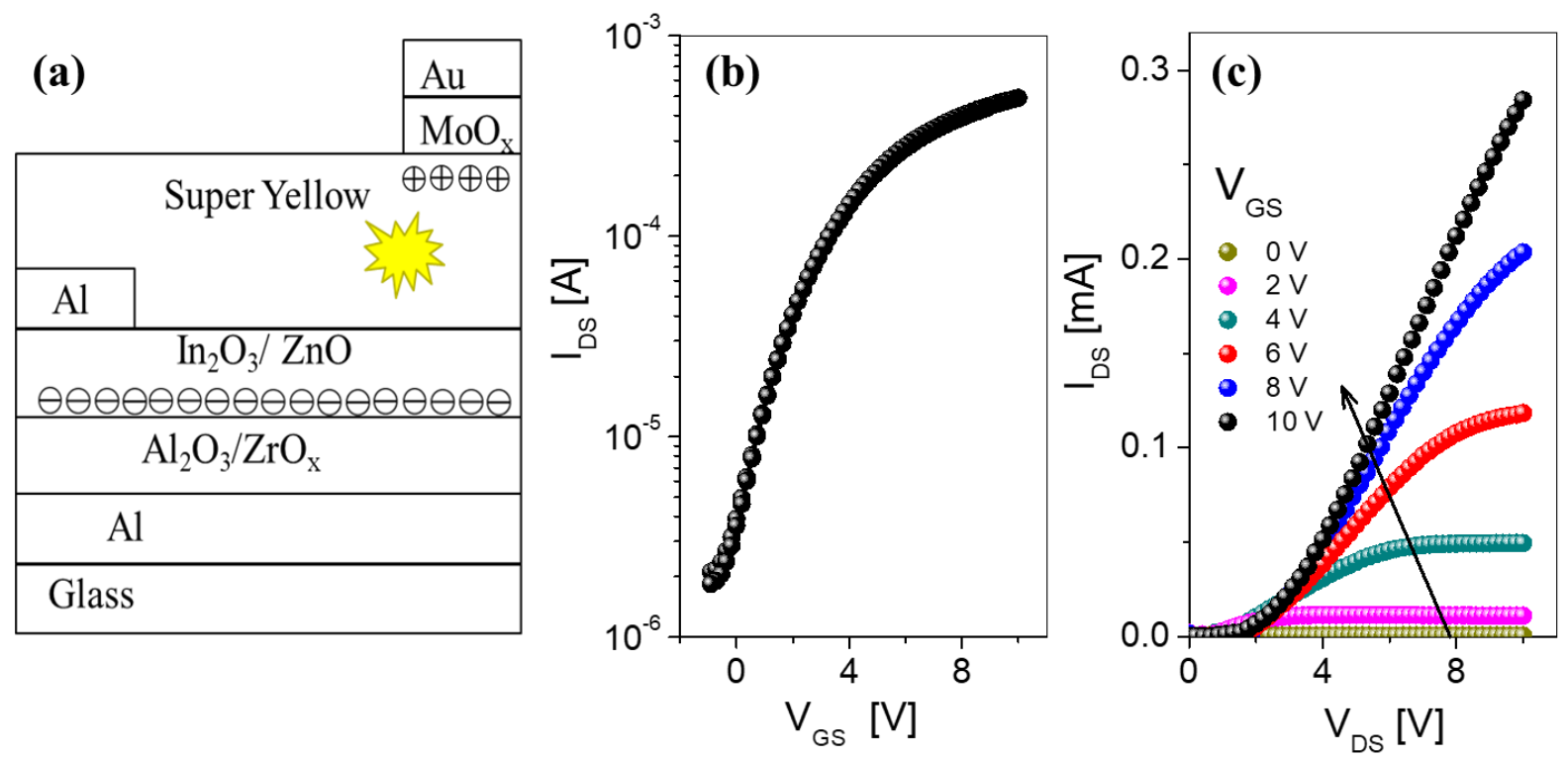

Figure 2. (a) Schematic HLET device structure comprised of light emitting polymer and solution processed dielectric and charge transport layers. (b) Electrical transfer and (c) Output characteristics of a HLET device.
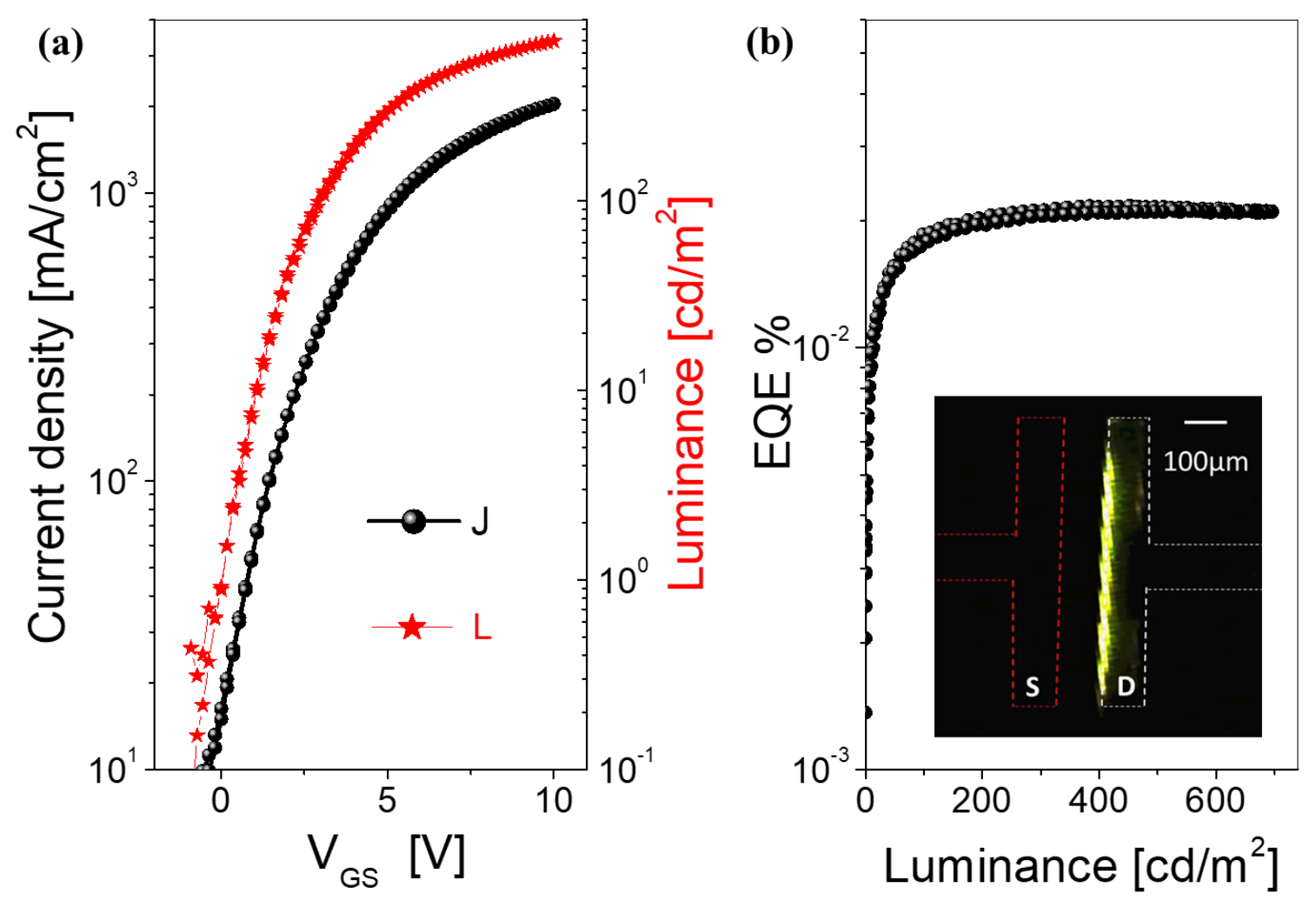

Figure 3. (a) Current density (J) and Luminance (L) as function of gate voltage. (b) External quantum efficiency of the HLET as a function of luminance; the inset shows an optical micrograph of the light emission emanating from the channel. The geometry of the buried source electrode is illustrated by the red dashed line. 
(a)

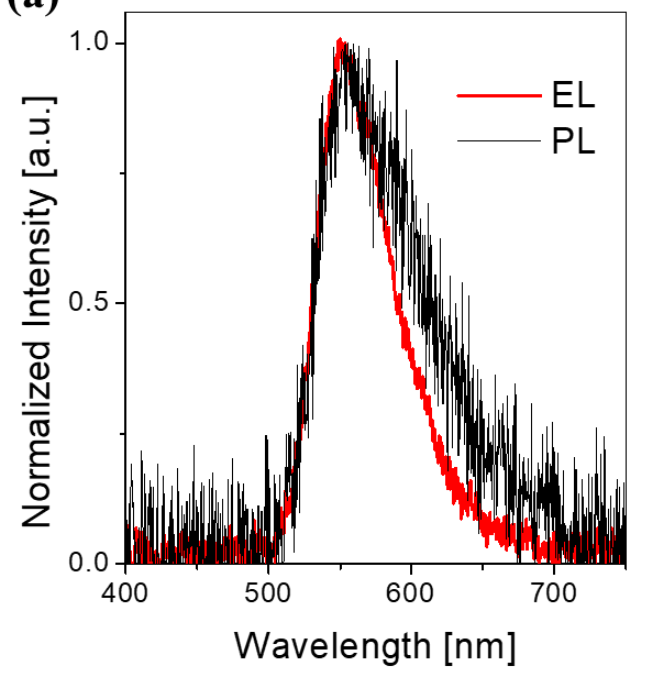

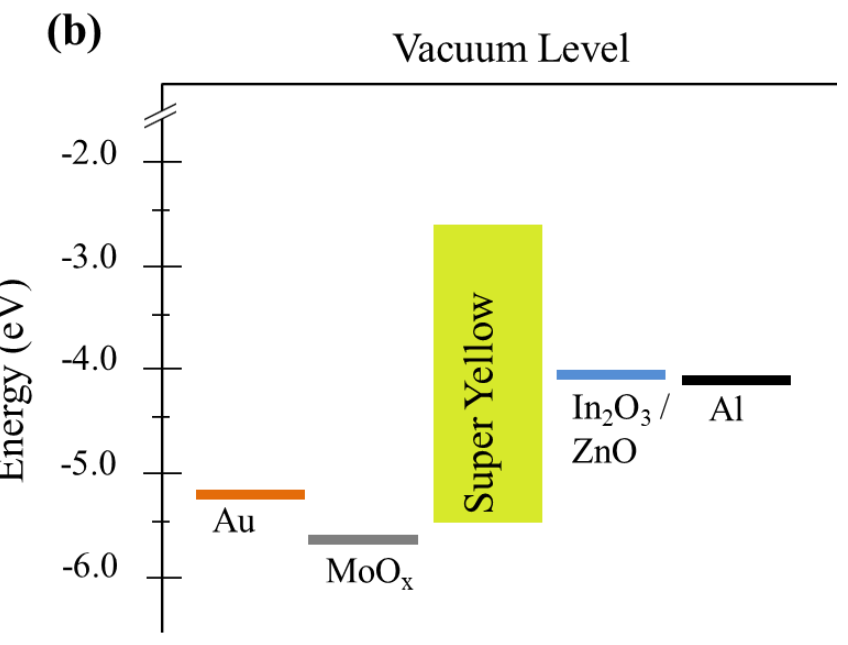

Figure 4. (a) Normalized photoluminescence (PL) and electroluminescence (EL) spectra for the HLET under optical and electrical excitation. (b) Schematic Energy level diagram for the materials used in the HLETs.

\section{References}

[1] Yu, X.; Marks, T. J.; Facchetti, A. Metal Oxides for Optoelectronic Applications. Nat. Mater. 2016, 15, 383-396.

[2] Banger, K. K.; Yamashita, Y.; Mori K.; Peterson, R. L.; Leedham, T.; Rickard, J.; Sirringhaus, H. Low-Temperature, High-Performance Solution-processed Metal Oxide ThinFilm Transistors Formed by a 'Sol-Gel on Chip' Process. Nat. Mater. 2011, 10, 45-50.

[3] Jung, H. Y.; Kang, Y.; Hwang, A. Y.; Lee, C. K.; Han, S.; Kim, D. H.; Bae, J. U.; Shin, W. S.; Jeong, J. K. Origin of the Improved Mobility and Photo-Bias Stability in a DoubleChannel Metal Oxide Transistor. Sci. Reports. 2014, 4, 3765.

[4] Park, J. C.; Lee, H. N. Improvement of the Performance and Stability of Oxide Semiconductor Thin-Film Transistors Using Double-Stacked Active Layers. IEEE Electron Device Letters. 2012, 33, 6, 818.

[5] Liu, G. X.; Liu, A.; Shan, F. K.; Meng, Y.; Shin, B. C.; Fortunato, E.; Martins, R. HighPerformance fully Amorphous Bilayer Metal-oxide Thin Film Transistors using Ultrathin Solution-Processed ZrOx Dielectric. Appl. Phys. Lett. 2014, 105, 113509.

[6] Ha, T. J.; Dodabalapur, A. Photo Stability of Solution-Processed Low-Voltage High Mobility Zinc-Tin-Oxide/ $\mathrm{ZrO}_{2}$ Thin-Film Transistors for Transparent Display Applications. Appl. Phys. Lett. 2013, 102, 123506. 
[7] Liu, A.; Liu, G. X.; Zhu, H. H.; Xu, F.; Fortunato, E.; Martins, R.; Shan, F. K. Fully Solution-Processed Low-Voltage Aqueous $\mathrm{In}_{2} \mathrm{O}_{3}$ Thin-Film Transistors Using an Ultrathin $\mathrm{ZrO}_{\mathrm{x}}$ Dielectric. ACS Appl. Mater. \&Int. 2014, 6, 17364-17369.

[8] Muhieddine, K.; Ullah, M.; Pal, B. N.; Burn, P. L.; Namdas, E. B. All Solution-Processed, Hybrid Light Emitting Field-Effect Transistors. Adv. Mater. 2014, 26, 6410-6415.

[9] Nakanotani, H.; Yahiro, M.; Adachi, C. Ambipolar Field-Effect Transistor Based on Organic-Inorganic Hybrid Structure. Appl. Phys. Lett. 2007, 90, 262104.

[10] Yamada, K.; Yamao, T.; Hotta. S. Light-Emitting Field-Effect Transistors Having Combined Organic Semiconductor and Metal Oxide Layers. Adv. Mater. 2013, 25, 2860.

[11] Muhieddine, K.; Ullah, M.; Maasoumi, F.; Burn, P. L.; Namdas, E.B. Hybrid AreaEmitting Transistors: Solution Processable and with High Aperture Ratios. Adv. Mater. 2015, 27, 6677-6682.

[12] Ullah, M.; Lin, Y. H.; Muhieddine, K.; Lo, S. C.; Anthopoulos, T. D.; Namdas, E. B. Hybrid Light-Emitting Transistors Based on Low-Temperature Solution-Processed Metal Oxides and a Charge-Injecting Interlayer. Adv. Opt. Mater. 2016, 4, 231-237.

[13] Kwon, S. J.; Han, T. H.; Kim, Y. H.; Ahmed, T.; Seo, H. K.; Kim, H.; Kim, D. J.; Xu, W.; Hong, B. H.; Zhu, J. X.; Lee, T. W. Solution-Processed n-Type Graphene Doping for Cathode in Inverted Polymer Light-Emitting Diodes. ACS Appl. Mater. \&Int. 2018, 10, 4874-4881.

[14] Kim, Y. H.; Han, T. H.; Cho, H.; Min, S. Y.; Lee, C. L.; Lee, T. W. Polyethylene Imine as an Ideal Interlayer for Highly Efficient Inverted Polymer Light-Emitting Diodes. Adv. Funct. Mater. 2014, 24, 3808-3814.

[15] Höfle, S.; Schienle, A.; Bruns, M.; Lemmer, U.; Colsmann, A. Enhanced Electron Injection into Inverted Polymer Light-Emitting Diodes by Combined Solution-Processed Zinc Oxide/Polyethylenimine Interlayers. Adv. Mater. 2014, 26, 2750-2754.

[16] Stadler, P.; Track, A. M.; Ullah, M.; Sitter, H.; Matt, G. J.; Koller, G.; Singh, T. B.; Neugebauer, H.; Sariciftci, N.S.; Ramsey, M. G. The Role of the Dielectric Interface in Organic Transistors: A Combined Device and Photoemission Study. Org. Electr. 2010, 11, 207-211.

[17] Tetzner, K.; Lin, Y. H.; Regoutz, A.; Seitkhan, A.; Payne, D. J.; Anthopoulos, T. D. Sub-Second Photonic Processing of Solution-Deposited Single Layer and Heterojunction Metal Oxide Thin-Film Transistors Using a High-Power Xenon Flash Lamp. J. Mater. Chem. C. 2017, 10.1039/C7TC03721J. 
[18] Labram, J. G.; Lin Y. H.; Anthopoulos, T. D. Exploring Two-Dimensional Transport Phenomena in Metal Oxide Heterointerfaces for Next-Generation, High-Performance, ThinFilm Transistor Technologies. Small, 2015, 11, 41, 5472-5482.

[19] Lin, Y. H.; Faber, H.; Labram, J. G.; Stratakis, E.; Sygellou, L.; Kymakis, E.; Hastas, N. A.; K. Zhao, R. Li; Amassian, A.; Treat, N. D.; McLachlan M.; Anthopoulos, T. D. High Electron Mobility Thin- Film Transistors Based on Solution- Processed Semiconducting Metal Oxide Heterojunctions and Quasi- Superlattices. Adv. Sci., 2, 2198 (2015).

[20] Tetzner, K.; Isakov, I.; Regoutz, A.; Paone D. J.; Anthopoulos, T. D. The impact of PostDeposition Annealing on the Performance of Solution-Processed Single Layer $\operatorname{In}_{2} \mathrm{O}_{3}$ and Isotype $\mathrm{In}_{2} \mathrm{O}_{3} / \mathrm{ZnO}$ Heterojunction Transistors. J. Mater. Chem. C. 2017, 5, 59.

[21] Faber, H.; Das, S.; Lin, Y. H.; Pliatsikas, N.; Zhao, K.; Kehagias, T.; Dimitrakopulos, G.; Amassian, A.; Patsalas, P. A.; Anthopoulos, T. D. Heterojunction Oxide Thin-Film Transistors with Unprecedented Electron Mobility Grown from Solution. Sci. Adv. 2017, 3, e1602640.

[22] Adamopoulos, G.; Thomas, S.; McLachlan, M.; Bradley, D.D.C.; Anthopoulos, T.D. High-Mobility Low-Voltage $\mathrm{ZnO}$ and Li-Doped $\mathrm{ZnO}$ Transistors Based on $\mathrm{ZrO}_{2}$ High-k Dielectric Grown by Spray Pyrolysis in Ambient Air. Adv. Mater. 2011, 23, 1894-1898.

[23] Tokmoldin, N.; Griffiths, N.; Bradley, D.D.C.; Haque, S.A. A Hybrid InorganicOrganic Semiconductor Light-Emitting Diode Using $\mathrm{ZrO} 2$ as an Electron-Injection Layer. Adv. Mater. 2009, 21, 3475-3478.

[24] Spreitzer, H.; Becker, H.; Kluge, E.; Kreuder, W.; Schenk, H.; Demandt, R.; Schoo, H. Soluble Phenyl-Substituted PPVs - New Materials for Highly Efficient Polymer LEDs. Adv. Mater. 1998, 10, 1340; Sundström, A.; Matyba, P.; Edman, L. Yellow-Green Light-Emitting Electrochemical Cells with Long Lifetime and High Efficiency. Appl. Phys. Lett. 2010, 96, 053303.

[25] Ullah, M.; Tandy, K.; Yambem, S. D.; Aljada, M.; Burn, P. L.; Meredith, P.; Namdas, E. B. Simultaneous Enhancement of Brightness, Efficiency, and switching in RGB Organic Light Emitting Transistors. Adv. Mater. 2013, 25, 6213.

[26] Ullah, M.; Armin, A.; Tandy, K.; Yambem, S. D.; Burn, P. L.; Meredith, P.; Namdas, E. B. Defining the Light Emitting Area for Displays in the Unipolar Regime of Highly Efficient Light Emitting Transistors. Sci. Rep. 2015, 5, 8818.

[27] Chaudhry, M. U.; Muhieddine, K.; Wawrzinek, R.; Li, J.; Lo, S-C.; Namdas, E. B. Nano-Alignment in Semiconducting Polymer Films: A Path to Achieve High Current Density 
and Brightness in Organic Light Emitting Transistors. ACS Photonics. 2018, doi: 10.1021/acsphotonics.8b00011.

[28] Choi, H. H.; Cho, K; Frisbie, C. D.; Sirringhaus, H.; Podzorov, V. Critical Assessment of Charge Mobility Extraction in FETs. Nat. Mater. 2018, 17, 2-7.

[29] Gwinner, M. C.; Khodabakhsh, S.; Song, M. H.; Schweizer, H.; Giessen, H.; Sirringhaus, H. Integration of a Rib Waveguide Distributed Feedback Structure into a Light-Emitting Polymer Field-Effect Transistor. Adv. Funct. Mater. 2009, 19, 1360-1370. 


\section{TOC}

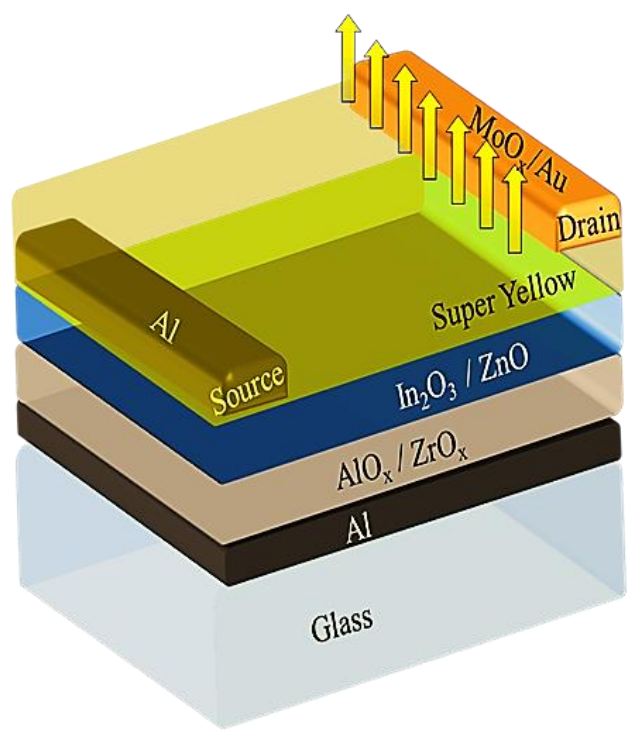

